\title{
Lysine and arginine reduce the effects of cerebral ischemic insults and inhibit glutamate-induced neuronal activity in rats
}

\author{
Takashi Kondoh ${ }^{1,2}$, Makiko Kameishi' ${ }^{1}$ Hruda Nanda Mallick ${ }^{3}$, Taketoshi Ono ${ }^{4}$ and Kunio Torii ${ }^{*}$ * \\ Institute of Life Sciences, Ajinomoto Co., Inc., Kawasaki, Japan \\ 2 AJINOMOTO Integrative Research for Advanced Dieting, Graduate School of Agriculture, Kyoto University, Kyoto, Japan \\ ${ }^{3}$ Department of Physiology, All India Institute of Medical Sciences, New Delhi, India \\ ${ }^{4}$ System Emotional Science, Graduate School of Medicine and Pharmaceutical Sciences, University of Toyama, Toyama, Japan
}

\section{Edited by:}

Mark Laubach, The John B. Pierce

Laboratory, USA; Yale University School

of Medicine, USA

\section{Reviewed by:}

Ivan E. de Araujo, The John B. Pierce Laboratory, USA; Yale University, USA

Tatsuya Abe, Oita Medical University, Japan;

Andras Hajnal, The Pennsylvania State University, USA

\section{*Correspondence:}

Kunio Torii, Institute of Life Sciences, Ajinomoto Co., Inc., Suzuki-cho 1-1, Kawasaki-ku, Kawasaki 210-8681, Japan.

e-mail:kunio_tori@@ajinomoto.com
Intravenous administration of arginine was shown to be protective against cerebral ischemic insults via nitric oxide production and possibly via additional mechanisms. The present study aimed at evaluating the neuroprotective effects of oral administration of lysine (a basic amino acid), arginine, and their combination on ischemic insults (cerebral edema and infarction) and hemispheric brain swelling induced by transient middle cerebral artery occlusion/reperfusion in rats. Magnetic resonance imaging and 2,3,5-triphenyltetrazolium chloride staining were performed 2 days after ischemia induction. In control animals, the major edematous areas were observed in the cerebral cortex and striatum. The volumes associated with cortical edema were significantly reduced by lysine $(2.0 \mathrm{~g} / \mathrm{kg})$, arginine $(0.6 \mathrm{~g} / \mathrm{kg})$, or their combined administration $(0.6 \mathrm{~g} / \mathrm{kg}$ each). Protective effects of these amino acids on infarction were comparable to the inhibitory effects on edema formation. Interestingly, these amino acids, even at low dose $(0.6 \mathrm{~g} / \mathrm{kg})$, were effective to reduce hemispheric brain swelling. Additionally, the effects of in vivo microiontophoretic (juxtaneuronal) applications of these amino acids on glutamateevoked neuronal activity in the ventromedial hypothalamus were investigated in awake rats. Glutamate-induced neuronal activity was robustly inhibited by microiontophoretic applications of lysine or arginine onto neuronal membranes. Taken together, our results demonstrate the neuroprotective effects of oral ingestion of lysine and arginine against ischemic insults (cerebral edema and infarction), especially in the cerebral cortex, and suggest that suppression of glutamate-induced neuronal activity might be the primary mechanism associated with these neuroprotective effects.

Keywords: Iysine, arginine, ischemia, middle cerebral artery occlusion, cerebral edema, glutamate receptor, magnetic resonance imaging, microiontophoretic application

\section{INTRODUCTION}

L-Arginine, a basic amino acid, is a precursor of nitric oxide (NO) that displays potent vascular relaxant properties. Intravenous administration of arginine (Morikawa et al., 1992, 1994) or NO donors (Zhang and Iadecola, 1994) have been shown to reduce ischemic infarction, while administration of NO synthase (NOS) inhibitors exacerbates brain damage (Kuluz et al., 1993). However, it must be noted that different lines of evidence suggesting that arginine exacerbates (Zhao et al. 2003) and NOS inhibitors ameliorate (Buisson et al., 1992; Kozniewska et al., 1995; Quast et al., 1995) ischemic infarction have also been reported.

In any event, arginine does not seem to be the only basic amino acid involved in neuroprotection against brain injury. Ebenezar et al. (2003) reported the protective effects of arginine and lysine combinations against experimentally induced myocardial infarction in rats. While sharing many common properties with $\mathrm{L}$-arginine, $\mathrm{L}$-lysine is a basic amino acid that does not function as a substrate for NO synthesis (Smulders et al., 1997). In addition, lysine is a potent inhibitor of arginase and hence blocks arginase-mediated urea synthesis from arginine (Egan et al., 1995). Intravenous infusion of lysine resulted in a significant increase in plasma levels of arginine (Kato et al., 1987), suggesting increased availability of arginine for $\mathrm{NO}$ synthesis. In general, lysine signaling might play a number of important physiological functions. In fact, inhibition of atherosclerosis formation (Yamori et al., 1978) and cellular proliferation properties (Datta et al., 2000) has been reported. Furthermore, lysine-enriched experimental foods suppress stress-induced anxiety in rats and humans (Smriga and Torii, 2003a; Smriga et al., 2004), an effect that was further enhanced by combination with arginine (Smriga and Torii, 2003b; Smriga et al., 2007). Voluntary ingestion of lysine in drinking water tends to reduce the incidence of stroke in stroke-prone spontaneously hypertensive rats (Yamori et al., 1977). To date, however, effects of lysine alone, or combination of lysine and arginine, on cerebral ischemic damages have not been investigated.

The role of the acidic amino acids, glutamate and aspartate, as excitatory neurotransmitters in the brain is well documented (Johnson, 1978).Compared to other amino acids, the concentrations 
of these acidic amino acids are consistently low in both circulation and cerebrospinal fluids, whereas concentrations of basic amino acids (lysine and arginine) are high (Nishimura et al., 1995). This phenomenon might result from the presence of high-affinity carrier systems for basic amino acids, resulting in high uptake of lysine and arginine into the brain (Oldendorf and Szabo, 1976). In any case, the physiological function of such high levels of basic amino acids in the brain remains unclear.

It is noticeable that, during ischemia/reperfusion, high levels of glutamate release followed by calcium overload and abnormal production of free radicals occurs (Won et al., 2002). The N-methyl-Daspartate (NMDA) receptor, the major type of excitatory glutamate receptor, comprises ligand-gated cation selective channel whose activity is blocked by divalent cation $\mathrm{Mg}^{2+}$ and $\mathrm{Zn}^{2+}$ (Mayer et al., 1984; Nowak et al., 1984). Accordingly, we have hypothesized that basic (and hence cationic) amino acids might suppress glutamateinduced neuronal activation and prevent neuronal death in the brain. If this hypothesis is confirmed, oral administration of basic amino acids may be effective to suppress ischemic insults. To our knowledge, there is no available evidence on the effects of basic amino acids on neuronal activity in vivo.

The present study aimed at investigating the neuroprotective effects of lysine, arginine, and their combination during the acute phase (2 days after the ischemia/reperfusion) of cerebral edema formation, hemispheric brain swelling and infarction as revealed by magnetic resonance imaging (MRI) and 2,3,5-triphenyltetrazolium chloride (TTC) histological staining in rats. Because cerebral edema generally accompanies with brain swelling and resulting in excessive intracranial pressure, finding ways to prevent cerebral edema has been a major research endeavor for reducing mortality or delayed neural injury after ischemia. We also examined the influence of microiontophoretically (juxtaneuronally) applied basic amino acids on glutamate-induced single unit activity in the ventromedial nucleus of the hypothalamus (VMH) neurons of awake rats. We undertook this study in conscious rats, as opposed to previous studies where the interactions between glutamate and test compounds were restricted to cultured neurons (Mayer et al., 1984; Nowak et al., 1984). The results of the present study suggest that the ingestion of some specific classes of amino acids may result in beneficial neuroprotective effects.

\section{MATERIALS AND METHODS}

We tested two main hypotheses, namely, both reduction of cerebral ischemic insults and inhibition of glutamate-induced neuronal activity by lysine and arginine administration in rats.

\section{ANIMALS}

In the ischemia experiments, 64 male Wistar rats (Charles River Laboratories Japan, Yokohama, Japan), weighing 280-320 g, were randomly assigned to five groups: saline group (control, $n=12$ ), $0.6 \mathrm{~g} / \mathrm{kg}$ lysine group $(n=14), 2.0 \mathrm{~g} / \mathrm{kg}$ lysine group $(n=13), 0.6 \mathrm{~g} /$ $\mathrm{kg}$ arginine group $(n=11)$, and combination of $0.6 \mathrm{~g} / \mathrm{kg}$ lysine and $0.6 \mathrm{~g} / \mathrm{kg}$ arginine group $(n=14)$. In the neuronal recording experiments, eight male Wistar rats, weighing 300-500 g, were used. Each rat was housed individually in a wire-meshed cage with free access to laboratory chow (CRF-1; Oriental Yeast, Tokyo, Japan) and tap water in a room where temperature $\left(23 \pm 1^{\circ} \mathrm{C}\right)$, relative humidity (50 $\pm 10 \%)$, and light (07:00-19:00) were controlled. All experiments were approved by the local committee on Ethics for Animal Experimentation and were carried out in accordance with the National Institute of Health Guide for the Care and Use of Laboratory Animals and with guiding principles for the Care and Use of Animals in the Field of Physiological Sciences recommended by the Physiological Society of Japan. All necessary actions were undertaken to minimize animal suffering and reduce the number of animals used.

\section{SURGERY FOR CEREBRAL ISCHEMIA}

After overnight fasting, rats were subjected to $1 \mathrm{~h}$ of middle cerebral artery (MCA) occlusion (MCAO) followed by reperfusion, a model of focal ischemia resembling that of human stroke (Belayev et al. 1996). The ischemic surgery (intraluminal occlusion technique) was as reported earlier (Kondoh et al., 2002; Torii et al., 2004). Briefly, rats were anesthetized with $1.5 \%$ halothane in a mixture of $30 \% \mathrm{O}_{2} / 70 \% \mathrm{~N}_{2} \mathrm{O}$ gas and maintained the rectal temperature at $37^{\circ} \mathrm{C}$ by a heating pad. An artificial embolus (a 4-0 Ethicon nylon filament with tip diameter tapered to the size of 25 or 24-gauge needle) was inserted from external carotid artery, via the internal carotid artery, to the base of either the left or right MCA to stop blood flow to the MCA. During ischemia, anesthesia was interrupted and rats were allowed to recover for $1 \mathrm{~h}$ and behavioral tests (see below) were performed to confirm successful unilateral ischemia. After $1 \mathrm{~h}$ of ischemia, the rats were re-anesthetized with halothane, the embolus withdrawn, the blood reperfusion established, and the skin sutured. After surgery, an antibiotic (gentamicine sulfate, Gentacin ${ }^{\circledast}$ Injection, Schering-Plough, Osaka, Japan) was administered systematically ( $8 \mathrm{mU}$, i.m.).

\section{SURGERIES FOR NEURONAL RECORDING}

Prior to the cranioplastic surgery, rats were acclimated by handling and accustomed to being placed into a small restraining cage that was constructed with stainless steel rods.

For neuronal recording in the $\mathrm{VMH}$, clanioplastic surgery and skull opening surgery were performed on the separate days as described earlier (Tamura et al., 2000). Twenty minutes after an injection of atropine $(0.1 \mathrm{mg} / \mathrm{kg}$, i.p. $)$, each rat was anesthetized with pentobarbital sodium (50 mg/kg, i.p.), and mounted on a stereotaxic apparatus with the skull inclination aligned to the bregma - lambda suture points. The cranium was exposed and five stainless steel screws (2-mm diameter) were threaded into holes in the skull to serve as anchors for cranioplastic acrylic. Stainless steel wires were soldered onto two screws to serve as ground. The cranioplastic acrylic was built up on the skull and molded around the conical ends of two sets of double stainless steel rods (fake ear bars) which had a single steel bar on one end and two bars on the other end. Once the cement had hardened, these bars were removed, leaving impression indentations on the double end on each side of the acrylic block. During subsequent surgery, training sessions, and recording sessions, the double end of these fake ear bars was pressed into the indentations in the acrylic block while the single end was inserted into the normal ear bar slot in the stereotaxic apparatus and rigidly attached to it. Hence, these fake ear bars functioned as regular ear bars but could also be used in awake animals because they did not involve painful insertion into 
ear canals. A short length of stainless steel tubing (27-gauge) was embedded in the cranioplastic acrylic near bregma to serve as a reference coordinate pin during chronic recording. After surgery, the antibiotic (gentamicine sulfate) was administered topically and systematically ( $8 \mathrm{mU}$, i.m.).

After recovery (7-10 days) from the cranioplastic surgery mentioned above and following recording training (2 weeks; see next section), the rats were reanesthetized (pentobarbital sodium, $40 \mathrm{mg} / \mathrm{kg}$, i.p.) and mounted in the stereotaxic apparatus using the fake ear bars. A hole (2- to 3-mm diameter) for chronic recording was drilled through the cranioplastic cap and underlying skull located over the intended recording site of the $\mathrm{VMH}(\mathrm{A},-2.3$ to -3.3 from bregma; L, 0.3-1.0; V, 8.0-10.0). The exposed dura matter was covered with hydrocortisone ointment (Rinderon-VG ${ }^{\circledast}$ ointment, Shionogi, Osaka, Japan). The hole was covered with sterile cotton and sealed with epoxy glue.

\section{TRAINING FOR NEURONAL RECORDING}

Following recovery from the cranioplastic surgery, the rats were placed on a 20- to 23-h water-deprivation regimen for training of neuronal recording. Each rat was placed in a restraining cage with the head fixed rigidly and painlessly in the stereotaxic apparatus by the fake ear bars. While restrained, rats had access to a spout from which they learned to take water. Rats were trained during increasingly longer periods, initially $1 \mathrm{~h}$ to eventually $4 \mathrm{~h}$ per day. After an adaptation period, most rats accepted the restraint for up to $4 \mathrm{~h}$ without manifesting distress. Throughout the training and recording periods, the rat was allowed to ingest $20 \mathrm{ml}$ of fluid per session in the restraining cage. If a rat failed to take a total volume of 20-ml fluid in the training, tap water was given when the rat was returned to its home cage so that the final volume of total intake was $20 \mathrm{ml}$.

\section{AMINO ACID ADMINISTRATION}

In the ischemia experiments, $\mathrm{L}$-lysine $\mathrm{HCl}$ and $\mathrm{L}$-arginine $\mathrm{HCl}$ (Ajinomoto, Tokyo, Japan) were dissolved in saline. The $\mathrm{pH}$ was 5.59-5.65 for lysine $\mathrm{HCl}$ solution, 5.66 for arginine $\mathrm{HCl}$ solution, and 5.59 for the mixture solution of lysine and arginine. They were administered twice orally ( $5 \mathrm{ml} / \mathrm{kg}$ for twice): just $30 \mathrm{~min}$ before MCAO and $10 \mathrm{~min}$ before reperfusion. Each administration was comprised of half of the final dose. Physiological saline $(0.15 \mathrm{M}$ $\mathrm{NaCl}, \mathrm{pH}$ was not adjusted) was administered similarly to the control group.

\section{BEHAVIORAL TEST}

To assess whether the ischemia procedure was successful, the forelimb akinesia and the spontaneous rotational tests were performed during MCAO (i.e., during 1-h embolism). Detailed description and specific criteria for each test have been reported elsewhere (Borlongan et al., 2000; Torii et al., 2004). Briefly, the forelimb akinesia test involved tail suspension of the animal observation of the positions of the forelimbs. Rats with MCAO demonstrate a characteristic forelimb posture: it is stretched out on the ischemic side while in the contralateral side it is clipped to their chest muscles. For the spontaneous rotational test, the rat was placed in a box made of Plexiglas and the direction of the rat's rotation was observed during a 5-min session.

\section{MAGNETIC RESONANCE IMAGING (MRI)}

The $\mathrm{T}_{2}$-weighted brain images of ischemic rats were acquired using an MRI system (SMIS, Guilford, UK) consisted of a 4.7 $\mathrm{T} / 400$ horizontal superconducting magnet equipped with actively shielded gradient coils $(73 \mathrm{mT} / \mathrm{m}, 26 \mathrm{~cm}$ of free inner diameter). We measured ischemic edema only at 2 days following the ischemic surgery because maximal cerebral water content (cerebral edema) is measured at 1-2 days after MCAO, after which the edema attenuates gradually (Hatashita et al., 1988; Toung et al., 2002). Rats were anesthetized with $1.5 \%$ isoflurane in a mixture of $30 \% \mathrm{O}_{2} / 70 \% \mathrm{~N}_{2} \mathrm{O}$ gas. Body temperature was controlled at $37^{\circ} \mathrm{C}$ by circulating water. Their heads were fixed firmly in a non-magnetic stereotaxic apparatus specially designed for MRI. A home-made volume coil (60-mm diameter) was used for both transmission and reception of radio frequency wave. For the evaluation of cerebral edema volume and hemispheric brain swelling, 15 consecutive coronal brain images were acquired using conventional $\mathrm{T}_{2}$-weighted multi-slice spin-echo pulse sequence. The parameters were: time of repetition $=6,000 \mathrm{~ms}$, echo time $=80 \mathrm{~ms}$, field of view $=38.4 \mathrm{~mm} \times 38.4 \mathrm{~mm}$, matrix $=128 \times 128$, slice thickness $=1.2 \mathrm{~mm}$, number of slices $=15$ with no gap, slice interleave $=4$, no average.

For generating $\mathrm{T}_{2}$ maps, the coronal plane at the level of bregma, in which the extent of edema was the greatest (Torii et al., 2004), was excited using multi-echo spin-echo pulse sequence. The parameters were: time of repetition $=2,500 \mathrm{~ms}$, basal echo time $=20$ $\mathrm{ms}$, echo train length $=6$, field of view $=38.4 \mathrm{~mm} \times 38.4 \mathrm{~mm}$, matrix $=128 \times 128$, slice thickness $=2.0 \mathrm{~mm}$, no average .

\section{2,3,5-TRIPHENYLTETRAZOLIUM CHLORIDE (TTC) STAINING}

After the completion of the MRI experiments, rats were anesthetized with pentobarbital sodium $(50 \mathrm{mg} / \mathrm{kg}$, i.p.) and sacrificed by cervical dislocation. The brain was quickly removed for TTC (Sigma-Aldrich, St. Louis, MO, USA) histological staining. Coronal brain slices of $1 \mathrm{~mm}$ were obtained by a brain slicer (VT1000S, Leica, Wetzlar, Germany) and incubated in 2\% TTC dissolved in $0.1 \mathrm{M}$ phosphate-buffered saline at $37^{\circ} \mathrm{C}$ for $30 \mathrm{~min}$ and then fixed by immersion in $10 \%$ phosphate-buffered formalin as reported by Bederson et al. (1986). Photographs of coronal sections were taken with a digital camera (COOLPIX950, Nikon, Tokyo, Japan). The unstained areas of the fixed brain sections were defined as infarcted.

\section{NEURONAL RECORDING AND IN VIVOMICROIONTOPHORESIS}

Extracellular single unit activities were recorded from the VMH of awake rats. Although spontaneous firing rates of $\mathrm{VMH}$ neurons in awake rats are very low (Tamura et al., 2000), our pilot experiments suggested the presence of many silent neurons in $\mathrm{VMH}$, whose activities could be evoked by microiontophoretic application of glutamate on the neuronal membrane recorded. The method of microiontophoresis is well established for investigating effects of various bioactive substances on recorded neurons in the brain (Curtis and Koizumi, 1961). With this technique, charged molecules or ions can be ejected without negligible injury. This approach has a merit of bypassing the major diffusion barriers and of limiting the potential site of chemical action to the recorded neuron. 
An eight-barreled glass micropipette assembly was used for microiontophoretic application of chemicals and an indwelling carbon fiber (7- $\mu \mathrm{m}$ diameter; Toray, Tokyo, Japan) protruding 15-20 $\mu \mathrm{m}$ from the central barrel was used for neuronal recording. The outer barrels were filled with monosodium L-glutamate (0.05 M, pH 8.5), L-lysine $\mathrm{HCl}(0.05 \mathrm{M}, \mathrm{pH} 6.0)$, L-arginine (0.05 M, $\mathrm{pH} 6.0)$, L-threonine $(0.05 \mathrm{M}, \mathrm{pH} 8.5)$ and $\mathrm{NaCl}(0.15 \mathrm{M})$. The $\mathrm{pH}$ of amino acid solutions were adjusted as described above by $0.1 \mathrm{~N} \mathrm{HCl}$ or $0.1 \mathrm{~N} \mathrm{NaCl}$. Two barrels were filled with $4 \mathrm{M} \mathrm{NaCl}$ for current balancing. The chemicals were ejected pseudo-randomly using current strength of 10-90 nA of appropriate polarity and for the duration of 30-60 s by an microiontophoretic apparatus (DIA Medical System, Tokyo, Japan). Intertrial intervals of at least 2 min were allowed for neurons recover from the effects of chemical. To exclude any artifacts (i.e., current effects), the null baseline neuronal responses to $\mathrm{Na}^{+}$and $\mathrm{Cl}^{-}$by application of either plus or minus current on a $\mathrm{NaCl}$ barrel, respectively, were verified during the initial trials. A neuronal response of more than $30 \%$ from the basal firing rate was considered as significant responses to the chemicals applied.

\section{CALCULATION OF CEREBRAL EDEMA VOLUME}

Volume of cerebral edema was calculated as follows:

Edema volume $=($ number of voxels in edema region $)$ $\times$ (voxel volume),

where the voxel volume was $0.108 \mathrm{~mm}^{3}(0.3 \mathrm{~mm} \times 0.3 \mathrm{~mm} \times 1.2 \mathrm{~mm})$. The criterion of voxels within the edema region was defined as voxels with hyperintensity of $20 \%$ relative to the corresponding anatomical structures in the contralateral hemisphere (Kondoh et al., 2002; Torii et al., 2004). Voxels that corresponded to the cerebroventricles were excluded carefully. The anterior-posterior position to bregma of each image was determined by a rat brain atlas (Paxinos and Watson, 1997).

\section{CALCULATION OF HEMISPHERIC BRAIN SWELLING}

Because of limited space in the cranium, cerebral edema is generally accompanied by swelling of brain tissue and, hence, associates with increased intracranial pressure, which contributes significantly to the amount of neural tissue damaged.

Brain swelling of ischemic hemisphere, as noted by movement of mid-sagittal line to contralateral non-ischemic hemisphere, was determined on $\mathrm{T}_{2}$-weighted coronal MRI images at the level of bregma as follows:

$\%$ Swelling $=(($ area of ischemic hemisphere $\times 2 /$ area of both left and right hemispheres) -1$) \times 100$

Position of mid-sagittal line was determined manually with the aid of hypointense area between the left and right hemispheres (see Figure 1).

\section{CALCULATION OF T ${ }_{2}$ VALUES}

$\mathrm{T}_{2}$ maps calculated from the multi-echo images were produced on the basis of pixel-by-pixel with the use of linear least-squares regression. Regions of interest for non-ischemic striatum and temporal muscle were selected manually on $\mathrm{T}_{2}$ maps and then the value was averaged.

\section{CALCULATION OF INFARCTION AREA}

The digital photographs of the coronal sections (color images) were converted to grayscale images and smoothed by a Gaussian filter with full-width at half maximum of 2.0 pixels. Infarct areas within each slice were extracted with a low cut filter, number of pixels was counted, and then calibrated. Total infarct area was calculated as the sum of infarct areas in each slice.

\section{STATISTICAL ANALYSIS}

Statistical comparisons between saline-treated control groups and amino acid-treated groups were made using one-way ANOVA followed by Fisher's protected least significant difference (PLSD) test. Differences were considered significant at $p<0.05$. Results are expressed as means \pm SEM.

\section{HISTOLOGY}

After the last recording session, each rat was anesthetized with pentobarbital sodium (50 mg/kg, i.p.). Several iron deposits were made stereotaxically around the recorded sites in the brain by passing positive current $(20 \mu \mathrm{A}, 30 \mathrm{~s})$ through a stainless steel electrode (0.2-mm diameter, polyurethane insulated except for $0.1 \mathrm{~mm}$ at the tip). Rats were then given a further overdose of anesthetic and perfused transcardially with heparinized $0.9 \%$ saline followed by $10 \%$ buffered formalin containing $2 \%$ potassium ferrocyanide. The brain was removed, and cut into $50-\mu \mathrm{m}$ coronal sections with a freezing microtome. Sections were stained with cresyl violet. All marking sites were verified microscopically. The location of each recording site was then calculated from the stereotaxic coordinates of the recording electrode and the coordinates of the marking electrodes.

\section{RESULTS}

\section{SUPPRESSION OF CEREBRAL EDEMA}

Volume of cerebral edema in anesthetized rats was measured by using high resolution MRI. Figure 1 shows five consecutive $\mathrm{T}_{2}$-weighted images in rats treated with $0.9 \%$ saline (control) or $2.0 \mathrm{~g} / \mathrm{kg}$ lysine. Increases in signal intensities (corresponding to cerebral edema) were observed clearly at 2 days following the MCAO/reperfusion surgery. In control rats, signal increase was found mainly in the cerebral cortex and striatum (caudate-putamen). In addition to the formation of cerebral edema, hemispheric brain swelling (the left hemisphere in the case of Figure 1A) was observed in the same animals. Oral administration of the high dose $(2.0 \mathrm{~g} / \mathrm{kg})$ of lysine suppressed cerebral edema, especially in cerebral cortex (Figure 1B). Brain swelling was also ameliorated following lysine treatment.

The total volume of cerebral edema was evaluated by calculating the total number of voxels corresponding to the edematous site throughout all image slices (Figure 2). The effect of lysine was dosedependent $[F(36,2)=3.685, p<0.05]$. In the $2.0 \mathrm{~g} / \mathrm{kg}$ lysine-treated group, total volume of cerebral edema was reduced significantly compared to the control group $\left(251.6 \pm 32.2 \mathrm{~mm}^{3}\right.$ in the control group and $153.8 \pm 23.0 \mathrm{~mm}^{3}$ in the $2.0 \mathrm{~g} / \mathrm{kg}$ lysine-treated group, $39 \%$ reduction, $p<0.05$ ). Administration of $0.6 \mathrm{~g} / \mathrm{kg}$ lysine alone $\left(196.0 \pm 32.5 \mathrm{~mm}^{3}, 22 \%\right.$ reduction, $\left.p=0.15\right)$ or $0.6 \mathrm{~g} / \mathrm{kg}$ arginine alone $\left(178.4 \pm 25.7 \mathrm{~mm}^{3}, 29 \%\right.$ reduction, $\left.p=0.08\right)$ tended to reduce the cerebral edema. However, combination of $0.6 \mathrm{~g} / \mathrm{kg}$ lysine and $0.6 \mathrm{~g} / \mathrm{kg}$ arginine $\left(162.6 \pm 21.0 \mathrm{~mm}^{3}\right)$ showed significant reduction 


\section{A Saline (Control)}

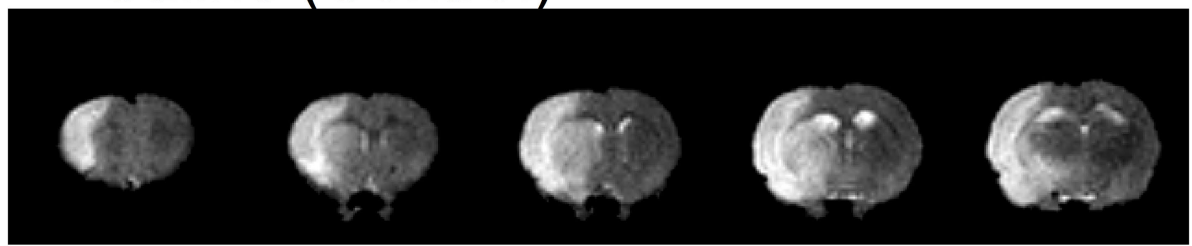

\section{в $\quad$ Lysine $(2.0 \mathrm{~g} / \mathrm{kg})$}

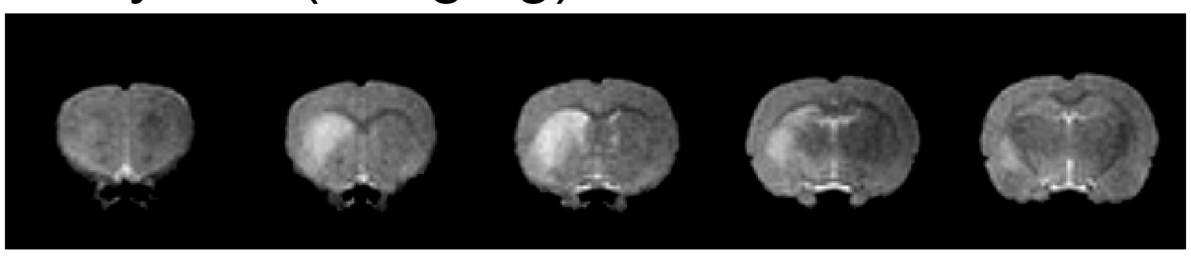

FIGURE 1 | Examples of five consecutive coronal brain images of MCAO rats administrated with (A) saline or (B) lysine as measured by $\mathrm{T}_{2}$-weighted MRI. Brain areas with hyperintensity (i.e., increase in water content) was observed in the left hemispheres, which represent presence of edema. Note that oral administration of lysine $(2.0 \mathrm{~g} / \mathrm{kg})$ suppressed cerebral edema, especially in the cerebral cortex. Lysine also reduced hemispheric brain swelling. Slice thickness, $1.2 \mathrm{~mm}$.

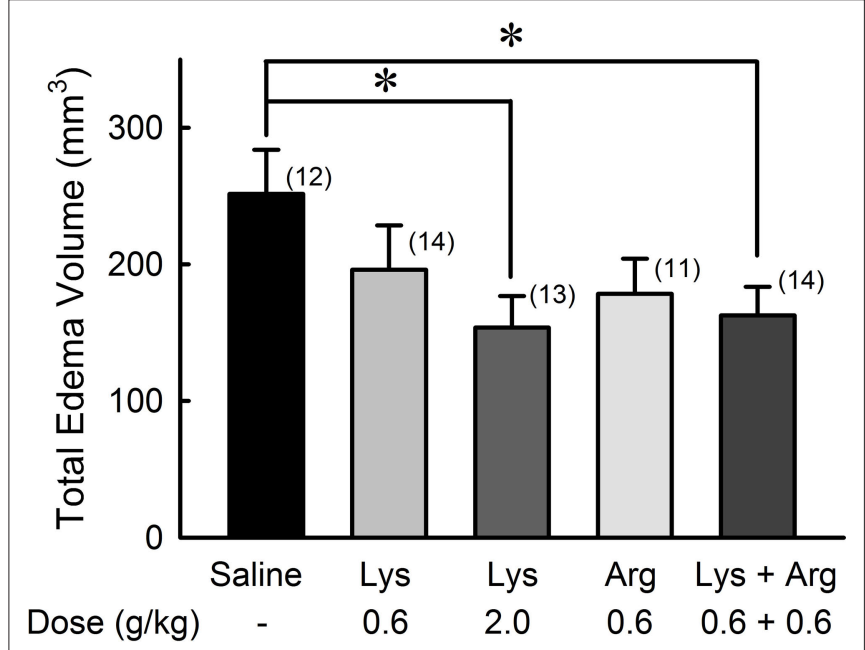

FIGURE 2 | Reduction of whole cerebral edema volume by oral administration of lysine (Lys) and/or arginine (Arg) in MCAO rats. Administration of lysine alone at high dose $(2.0 \mathrm{~g} / \mathrm{kg})$, or combined administration of lysine and arginine at low doses $(0.6 \mathrm{~g} / \mathrm{kg}$ each) significantly suppressed whole cerebral edema volume. Numbers between parentheses represent number of experiments. ${ }^{*} p<0.05$, significance compared to saline-treated control groups by ANOVA followed by Fisher's PLSD test.

of cerebral edema by $35 \%$ compared to the control group $(p<0.05)$. No significant differences were detected among $0.6 \mathrm{~g} / \mathrm{kg}$ lysine, $0.6 \mathrm{~g} / \mathrm{kg}$ arginine, and their combination groups.

The suppression of cerebral edema by oral administration of lysine or arginine was prevalent in the cerebral cortex (Figure 3). Administration of $2.0 \mathrm{~g} / \mathrm{kg}$ lysine, $0.6 \mathrm{~g} / \mathrm{kg}$ arginine, and a mixture of lysine and arginine resulted in significant reduction in cortical edema $(p<0.05)$, while no effect was observed on striatal edema in any amino acid groups. The protective effect of lysine on cortical

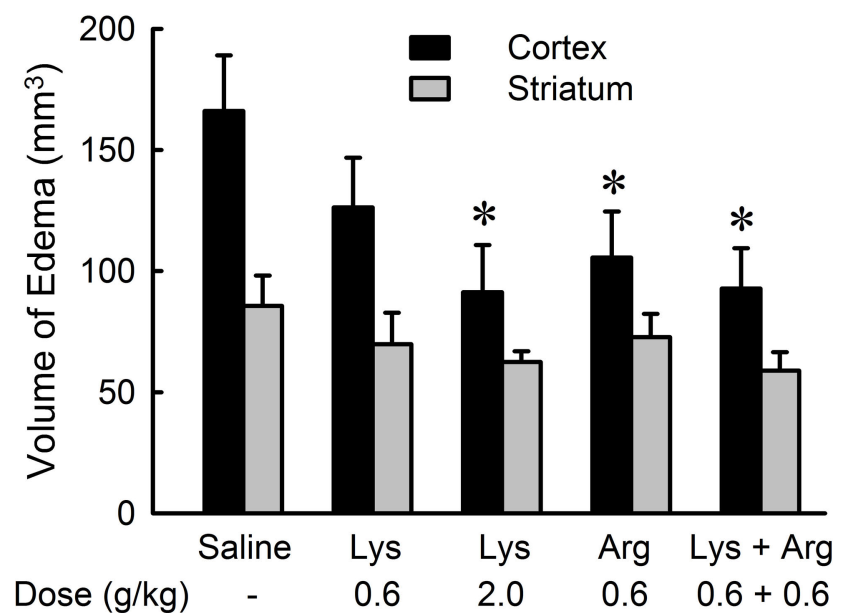

FIGURE 3 | Effects of lysine (Lys) and/or arginine (Arg) on cortical and striatal edema in MCAO rats. Note that significant reduction of edema volume was observed in the cerebral cortex but not in the striatum by administration of lysine $(2.0 \mathrm{~g} / \mathrm{kg})$, arginine $(0.6 \mathrm{~g} / \mathrm{kg})$, or combined administration of lysine and arginine. ${ }^{*} p<0.05$, significance compared to the saline-treated control group by ANOVA followed by Fisher's PLSD test.

edema was dose-dependent $[F(36,2)=4.758, p<0.05]$. There were no significant differences among $0.6 \mathrm{~g} / \mathrm{kg}$ lysine, $0.6 \mathrm{~g} / \mathrm{kg}$ arginine, and their combination groups.

To assess the effects of lysine and/or arginine with respect to the anteroposterior axis, extent of edema volume in each image slice (1.2-mm thickness) was calculated (Figure 4). In saline-treated controls, edema volume distributed across a wide area between +6 $\mathrm{mm}$ (anterior) to $-7.2 \mathrm{~mm}$ (posterior), with a peak at $-1.2 \mathrm{~mm}$ from bregma. Treatment of lysine or arginine appeared to reduce the edema volume across a large brain area, with a peak at $0 \mathrm{~mm}$ to bregma. 


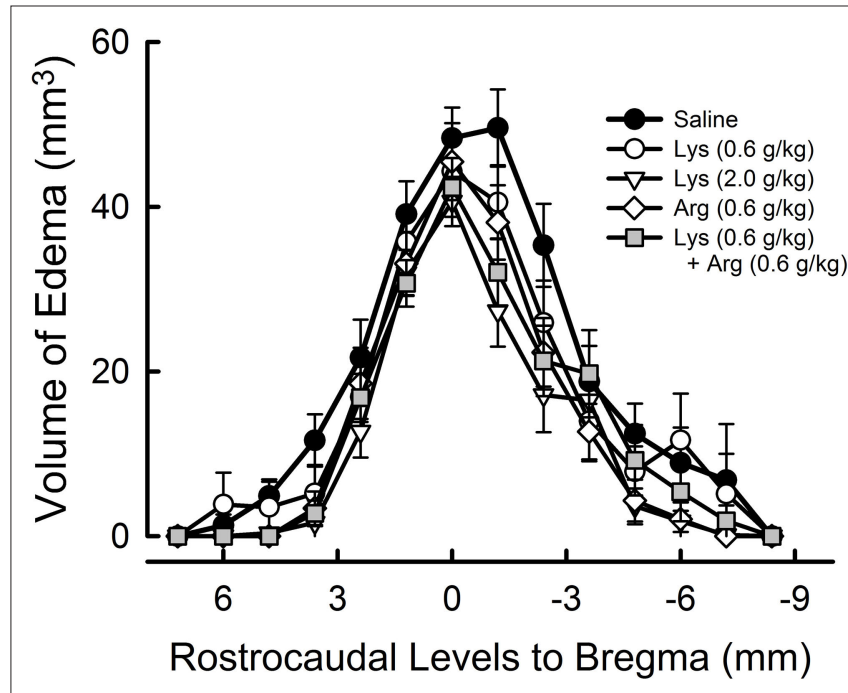

FIGURE 4 | Rostrocaudal extension of cerebral edema. The value 0 represents the bregma position in the antero-posterior axis, with more anterior parts represented by positive values and more posterior parts represented by negative values. Note that the extent of cerebral edema appeared to be reduced in overall levels by administration of lysine (Lys), arginine (Arg), or their combination.

Increases in tissue water content are known to associate with the increase in $T_{2}$ relaxation time ( $T_{2}$ value). To exclude any nonspecific effects of amino acid themselves on MRI signal intensities of non-ischemic regions, $\mathrm{T}_{2}$-weighted multi-echo images were acquired with six echo times (20, 40, 60, 80, 100, and $120 \mathrm{~ms}$ ). In the non-ischemic striatum and temporal muscle (an example of the extracerebral tissue), $\mathrm{T}_{2}$ values were approximately 70 and $38 \mathrm{~ms}$, respectively (Table 1). No amino acids altered $\mathrm{T}_{2}$ values in these non-ischemic regions (ANOVA, $p>0.05$ ), suggesting that neither lysine nor arginine modified MRI signal intensities in a non-specific manner.

\section{SUPPRESSION OF HEMISPHERIC BRAIN SWELLING}

Hemispheric brain swelling at the level of bregma was significantly reduced by administration of lysine and/or arginine compared with saline (Figure 5). Extents of swelling were $8.14 \pm 1.16 \%$ in the saline group, $5.14 \pm 0.81 \%$ in the $0.6 \mathrm{~g} / \mathrm{kg}$ lysine group $(p<0.05), 3.60 \pm 0.56 \%$ in the $2.0 \mathrm{~g} / \mathrm{kg}$ lysine group $(p<0.01)$, $4.36 \pm 1.05 \%$ in the $0.6 \mathrm{~g} / \mathrm{kg}$ arginine group $(p<0.01)$, and $3.57 \pm 0.71 \%$ in the combined administration of $0.6 \mathrm{~g} / \mathrm{kg}$ lysine and $0.6 \mathrm{~g} / \mathrm{kg}$ arginine $(p<0.01)$. The effect of lysine was dosedependent $[F(36,2)=6.845, p<0.01]$. No significant differences were detected among $0.6 \mathrm{~g} / \mathrm{kg}$ lysine, $0.6 \mathrm{~g} / \mathrm{kg}$ arginine, and their combination groups.

\section{SUPPRESSION OF CEREBRAL INFARCTION}

2,3,5-Triphenyltetrazolium chloride-staining method applied to the treated brain slices (Figure 6) revealed that the neuroprotective effects of lysine or arginine on cerebral infarction appeared to be similar to those observed with MRI methods. The effect of lysine was dose-dependent $[F(36,2)=3.543, p<0.05]$. Administration of $2.0 \mathrm{~g} / \mathrm{kg}$ lysine alone or combined administration of $0.6 \mathrm{~g} / \mathrm{kg}$ lysine
Table $1 \mid \mathrm{T}_{2}$ values in non-ischemic striatum and temporal muscle.

\begin{tabular}{llll}
\hline & \multicolumn{2}{c}{$\mathbf{T}_{2}$ values (ms) } \\
\cline { 2 - 3 } Oral administration & Striatum & $\begin{array}{l}\text { Temporal } \\
\text { muscle }\end{array}$ & $\boldsymbol{n}$ \\
\hline Saline & $69.0 \pm 1.27$ & $38.0 \pm 0.98$ & 12 \\
Lysine $(0.6 \mathrm{~g} / \mathrm{kg})$ & $68.8 \pm 0.84$ & $38.6 \pm 0.93$ & 14 \\
Lysine $(2.0 \mathrm{~g} / \mathrm{kg})$ & $71.6 \pm 1.45$ & $39.6 \pm 0.88$ & 13 \\
Arginine $(0.6 \mathrm{~g} / \mathrm{kg})$ & $70.4 \pm 1.07$ & $37.8 \pm 0.69$ & 11 \\
Lysine $(0.6 \mathrm{~g} / \mathrm{kg})+$ Arginine & $71.7 \pm 0.81$ & $38.4 \pm 0.67$ & 14 \\
$(0.6 \mathrm{~g} / \mathrm{kg})$ & & & \\
\hline
\end{tabular}

The ' $n$ ' represents numbers of experiments.

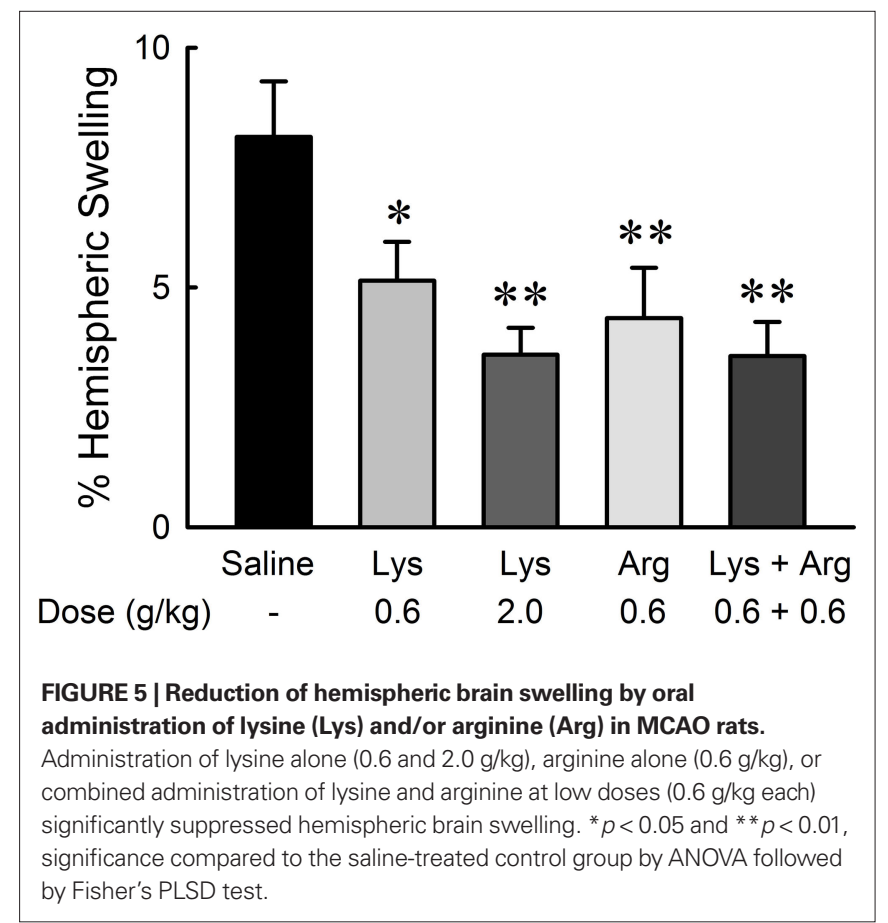

and $0.6 \mathrm{~g} / \mathrm{kg}$ arginine showed significant reductions of infarct areas compared to controls $(p<0.05)$. Administration of $0.6 \mathrm{~g} / \mathrm{kg}$ lysine alone or $0.6 \mathrm{~g} / \mathrm{kg}$ arginine alone tended to reduce infarct area. No significant differences were detected among $0.6 \mathrm{~g} / \mathrm{kg}$ lysine, $0.6 \mathrm{~g} /$ $\mathrm{kg}$ arginine, and their combination groups.

Infarct area measured by TTC staining was positively correlated with cerebral edema measured by MRI $(Y=76.8+1.100 X$, $r=0.736, p<0.0001)$ (Figure 7).

\section{SUPPRESSION OF GLUTAMATE-INDUCED ACTIVITY IN THE VMH}

We investigated the effects of lysine and arginine on glutamateinduced VMH neuronal activity in awake rats. Of a total of 25 VMH neurons recorded, 11 (44\%) neurons were inhibited by lysine and eight (32\%) neurons were inhibited by arginine (Table 2 ). No neurons showed any response to microiontophoretic applications of threonine, $\mathrm{Na}^{+}$and $\mathrm{Cl}^{-}$. The neuronal recording sites within the VMH were histologically confirmed. 


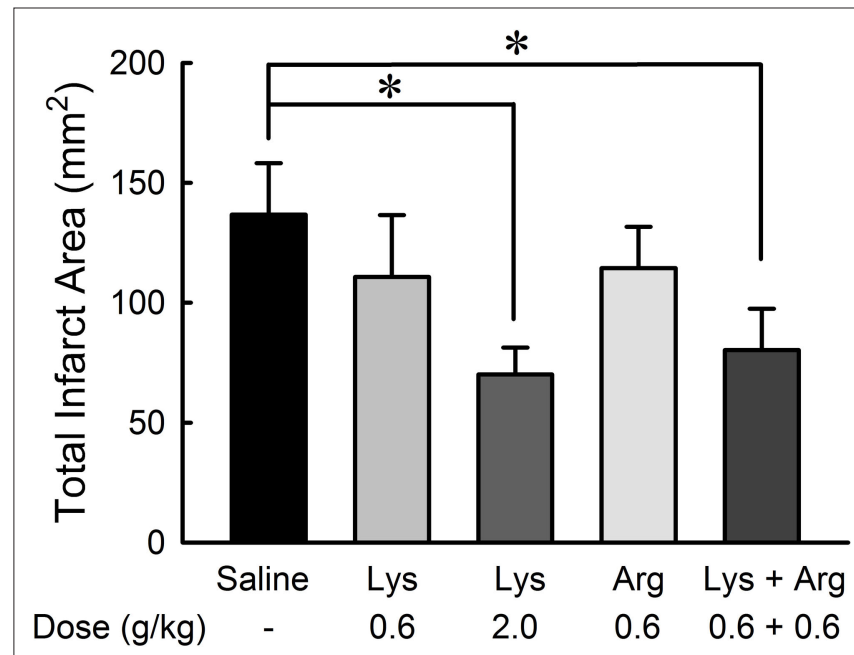

FIGURE 6 | Reduction of infarct area by oral administration of lysine (Lys) and/or arginine (Arg) in MCAO rats. Infarct area was measured with TTC staining. Note that administration of lysine alone at high dose $(2.0 \mathrm{~g} / \mathrm{kg})$ or combined administration of lysine and arginine at low dose $(0.6 \mathrm{~g} / \mathrm{kg})$ significantly suppressed infarct in consistent to the effects on cerebral edema as shown in Figure 2. ${ }^{*} p<0.05$, significance compared to the saline-treated control group by ANOVA followed by Fisher's PLSD test.

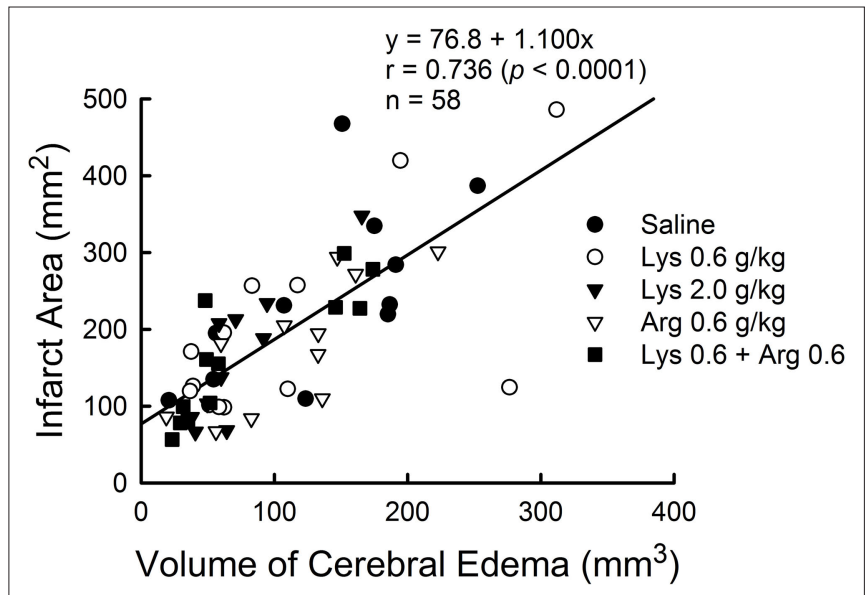

FIGURE 7 | Positive correlation between cerebral edema measured by MRI and infarct area measured by TTC staining in MCAO rats. Data from all experimental groups were combined for analysis. $Y=76.8+1.100 X$, $r=0.736, p<0.0001$.

As shown in Figure 8, the neuron shows little firing activity before application of glutamate. The neuronal activity of 3-10 spikes/s was evoked after the continuous application of glutamate $(-10$ or $-20 \mathrm{nA})$ to the neuron. Application of neither $\mathrm{Na}^{+}$nor $\mathrm{Cl}^{-}$evoked measurable responses. When lysine or arginine $(+50$ to $+70 \mathrm{nA})$ was applied to the neuronal membrane, glutamate-induced neuronal activity was completely suppressed, typically within $300 \mathrm{~ms}$. These inhibitory effects reversed gradually within 1-2 min, with activity increasing to preadministration levels after cessation of the application. Thus the inhibitory effects of lysine and arginine were reversible and reproducible. The inhibition by lysine appeared to persist for longer than by arginine.
Table 2 | Responsiveness of VMH neurons to microiontophoretically applied chemicals in awake rats.

Number of neurons

\begin{tabular}{lllll}
\cline { 2 - 3 } Chemicals & Excitatory & Inhibitory & No response & Total \\
\hline L-Lysine & $0(0 \%)$ & $11(44 \%)$ & $14(56 \%)$ & $25(100 \%)$ \\
L-Arginine & $0(0 \%)$ & $8(32 \%)$ & $17(68 \%)$ & $25(100 \%)$ \\
L-Threonine & $0(0 \%)$ & $0(0 \%)$ & $13(100 \%)$ & $13(100 \%)$ \\
$\mathrm{Na}^{+}$ & $0(0 \%)$ & $0(0 \%)$ & $25(100 \%)$ & $25(100 \%)$ \\
$\mathrm{Cl}^{-}$ & $0(0 \%)$ & $0(0 \%)$ & $25(100 \%)$ & $25(100 \%)$
\end{tabular}

Numbers in parentheses denote the percentage of neurons observed in each response.

\section{DISCUSSION}

Strategies for reducing cerebral edema in the early phase of ischemia play a fundamental role in the attempt to minimize subsequent chronic neuronal damage in stroke. The present study has demonstrated, for the first time, that oral administrations of high dose of lysine alone $(2.0 \mathrm{~g} / \mathrm{kg})$ or low dose of lysine and arginine in combination $(0.6 \mathrm{~g} / \mathrm{kg}$ each $)$, are both effective against experimental ischemic damages (i.e., cerebral edema and infarction) in a rat model of MCAO/reperfusion. These amino acids robustly suppressed edema in cortex but failed to do so in striatum. Interestingly, administration of these basic amino acids, even at low dose $(0.6 \mathrm{~g} / \mathrm{kg})$, significantly reduced hemispheric brain swelling. Furthermore, both lysine and arginine suppressed glutamateevoked neuronal activity, which may contribute to the reduction of excitotoxicity caused by excess glutamate released into the synapse during ischemia.

The present study aimed at demonstrating potential beneficial effects of lysine and arginine on ischemic insults. Since we expected moderate to weak effects of these amino acids by oral administration, administrations were made twice, before and during the ischemia. Therefore, we could not conclude whether the effects of lysine and arginine were prophylactic or therapeutic for ischemic cerebral damages. Further studies will be required to resolve this issue.

\section{SUPPRESSION OF GLUTAMATE-INDUCED NEURONAL ACTIVITY}

Lysine and arginine are positively charged amino acids, while glutamate is negatively charged. Both lysine and arginine completely blocked glutamate-evoked neuronal excitation in microiontophoresis experiments, suggesting that lysine and arginine suppress glutamatergic neuronal activity in vivo and dietary levels of these amino acids might modulate brain activity via glutamatergic inhibition. Glutamate activates ionotropic glutamate receptors, including NMDA receptor that behaves as a single-occupancy channel for small monovalent and divalent permeant cations (Zarei and Dani, 1994). Considering the rapid onset of the suppressive effects, lysine/arginine might block directly the ion channels (open channel block) via mechanisms similar to those associated with $\mathrm{Zn}^{2+}$ and $\mathrm{Mg}^{2+}$ (Mayer et al., 1984; Nowak et al., 1984). Another possibility relates to indirect actions of lysine/arginine via activation of non-glutamate receptors or via adjacent inhibitory interneurons. For example, lysine interacts directly with a variety of receptors and can act both as an 


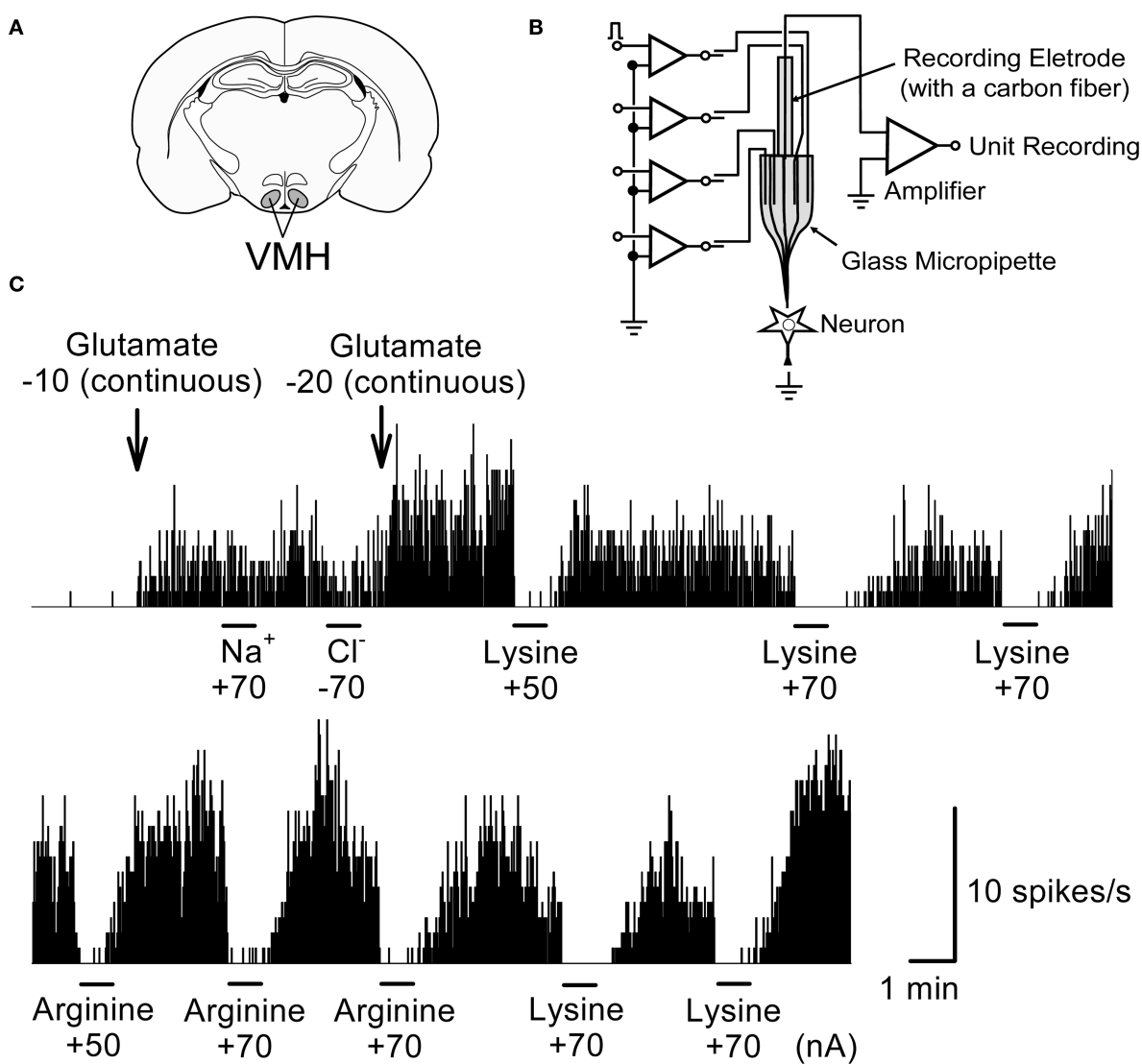

FIGURE 8 |An example of inhibitory responses to lysine and arginine applied microiontophoretically on glutamate-induced single unit activity in a rat $\mathrm{VMH}$ neuron. (A) The position of $\mathrm{VMH}$ in the rat brain. (B) A schematic representation of the multi-barreled glass micropipette with a recording electrode. (C) Suppression of glutamate-induced activity by lysine and arginine. Firing rate of this neuron was little at the basal level; therefore glutamate $(-10$ or $-20 \mathrm{nA})$ was applied continuously in the background to evoke steady-state levels of firing activity. Microiontophoretic applications (45 s) of either lysine or arginine did inhibit this glutamate-induced neuronal activity whereas neither $\mathrm{Na}^{+}$nor $\mathrm{Cl}^{-}$modified it. Lower trace was the continuous recording of the upper trace. Note that inhibitory responses by lysine and arginine occurred rapidly while the recovery of inhibitory responses occurred slowly for 1-2 min after cessation of the application. Numerical values under the chemicals represent current strength $(n A)$ applied for ejection of each chemical from the tip of multi-barreled micropipettes. The similar responses were repeatedly observed in other neurons. Ordinate: Neuronal discharge rate/s. Abscissa: time. Bars: period of microiontophoretic applications. partial antagonist of the serotonin receptor type 4 (Smriga and Torii, 2003a) and as an agonist of benzodiazepine (Chang and Gao, 1995), calcium-sensing (Conigrave et al., 2000) and “GPRC6A" (G-proteincoupled receptor, family $\mathrm{C}$, group 6, subtype A; Wellendorph et al., 2005) receptors. However, these mechanisms were less likely since microiontophoretically applied chemicals can act only within a very narrow area, possibly involving only the recorded neuron. Although it is premature to assign any particular function, our findings bring to light a functional interaction between basic amino acids and glutamate in the brain. Suppression of glutamatergic neurons by lysine and arginine might contribute to the suppression of cerebral ischemic damages. Further studies are needed to clarify the mechanisms of these inhibitory actions and potential therapeutic interventions.

\section{CEREBRAL CORTEX VS. STRIATUM}

Cerebral edema and infarction were reduced significantly in the cerebral cortex, but not in the striatum, after treatment with lysine alone, or with a combination of lysine and arginine. These differential alterations have also been reported following oral administration of melatonin, a pineal secretory product synthesized from tryptophan. For example, melatonin decreases the area of cortical infarction by $\sim 60 \%$ and striatal infarction by $30 \%$ (Borlongan et al., 2000) and decreases the cortical edema volume by $\sim 60 \%$ and striatal edema by $34 \%$ (Torii et al., 2004). The differential alterations of basic amino acids may be explained by the anatomical differences in blood vessel distribution, in which moderate but significant increases in bypass blood flow (collateral circulation) in the cerebral cortex compared with striatum. For example, arginine administration increases cerebral blood flow in ischemic conditions (Dalkara et al., 1994; Sadoshima et al. 1997). Intravenous arginine infusions increase cerebral blood volume in the cortex but not in the striatum in non-ischemic rats (Caramia et al., 1998).

Alternatively, higher number of cortical astrocytes (White et al., 1992) than striatal astrocytes (Bronstein et al., 1995) may account for better protection of the cortex than the striatum against ischemic insult. Astrocytes can exert protective actions on neural survival by releasing many neurotrophic factors, siphoning excess extracellular water and potassium ion, and reducing glutamate toxicity by removing glutamate from synapses (White et al., 1992; Ridet et al., 1997). Co-culture of astrocyte and neuron appear to promote higher levels 
of neuronal survival compared to purely neuronal cultures (Ridet et al., 1997). Glial cells were more vulnerable than neurons against ischemia (Borlongan et al., 2000). These findings suggest that glial cells are critical to neuronal survival and hence lysine and arginine might enhance survival of both neuronal and glial cells.

\section{ARGININE AND NEUROPROTECTION}

Protective effects of arginine against ischemic damages have been studied during the last 20 years by many researchers in terms of the "arginine-NO production-vasodilation" hypothesis. For example, intravenous administration of arginine increases cerebral blood flow both in baseline (Morikawa et al., 1992; Sadoshima et al., 1997; Caramia et al., 1998) and ischemic conditions (Dalkara et al., 1994; Sadoshima et al. 1997). Recovery of cerebral blood flow for more than $30 \%$ is associated with signal reappearance in electroencephalograms (Dalkara et al., 1994). The D-form of arginine (Morikawa et al., 1992; Dalkara et al., 1994) and the non-selective smooth muscle relaxant papaverine (Zhang and Iadecola, 1994) produce no such effects, suggesting that the observed actions are induced specifically by the L-form of arginine. Administration of NO donors such as sodium nitroprusside and 3-morpholinosydnonimine produce effects similar to those of arginine (Zhang and Iadecola, 1994), while administration of NOS inhibitors exacerbates ischemic damages (Kuluz et al., 1993). NO acts neuroprotector via $S$-nitrosylation of NMDA receptor thiol and suppression of NMDA receptor activity in the presence of conditions leading to the synthesis of nitrosonium ion $\left(\mathrm{NO}^{+}\right)$(Satoh and Lipton, 2007).

In contrast to the protective effects mentioned above, different lines of evidence suggest that arginine exacerbates ischemic infarction (Zhao et al. 2003) and NOS inhibitors ameliorate it (Buisson et al., 1992; Kozniewska et al., 1995; Quast et al., 1995). One plausible explanation for such discrepancy may be that the arginine effect depends on the redox state of the body (Lipton et al., 1993). For example, under conditions of preferential production of the NO radical (NO), arginine is cytotoxic via production of toxic free radicals, such as peroxynitrite anion $\left(\mathrm{ONOO}^{-}\right)$, which lead to indiscriminate damage to biological macromolecules including DNA, proteins, and membrane lipids (Halliwell et al., 1992; Lewén et al., 2000). The redox versatility of NO may explain its interconversion from neuroprotective to neurotoxic species by a change in the ambient milieu.

\section{REFERENCES}

Bederson, J. B., Pitts, L. H., Germano, S. M., Nishimura, M. C., Davis, R. L., and Bartkowski, H. M. (1986). Evaluation of 2,3,5-triphenyltetrazolium chloride as a stain for detection and quantification of experimental cerebral infarction in rats. Stroke 17, 1304-1308.

Belayev, L., Alonso, O. F., Busto, R., Zhao, W., and Ginsberg, M. D. (1996). Middle cerebral artery occlusion in the rat by intraluminal suture. Neurological and pathological evaluation of an improved model. Stroke 27, 1616-1623.

Borlongan, C. V., Yamamoto, M., Takei, N., Kumazaki, M., Ungsuparkorn, C., Hida, H., Sanberg, P. R., and Nishino,

Taken together, actions of arginine (and NO) are complex and the observed results may largely depend on a variety of factors, such as tissue regions, concentration and timing of $\mathrm{NO}$ production, and redox state of the body.

\section{LYSINE AND NEUROPROTECTION}

Although L-lysine shares many of the chemical properties of arginine (Smulders et al., 1997), effects of lysine on cerebral ischemic injury have not been investigated previously. We have demonstrated for the first time that oral administration of lysine alone or in combination with arginine is effective against cerebral ischemic insults. The effectiveness of lysine is of interest with respect to the following three issues: (1) lysine is not a precursor for NO synthesis, (2) the mechanism of neuroprotection may be distinct from the one used by arginine, and (3) $\mathrm{L}$-lysine is the limiting amino acid in proteins of feed grains such as wheat and corn (Torii et al., 1987) and therefore the risk of lysine deficiency is high in low socioeconomic human populations who depend predominantly on wheat for their protein supply (Young and Pellett, 1990). Therefore, deficiency of dietary lysine might increase the risk of stroke and hence fortification of lysine on foods might be beneficial for preventing ischemic insults. Additionally, combined ingestion of lysine with arginine may enhance the neuroprotective effects. Suppression of stress-induced anxiety has been shown to be enhanced by a combination of lysine and arginine in rats and humans (Smriga and Torii, 2003b; Smriga et al., 2007).

\section{CONCLUSION}

In summary, oral administration of high doses of lysine or combination of low dose of lysine with arginine is effective against cerebral edema and infarction caused by ischemia/reperfusion in a rat model of transient focal ischemia. Lysine and arginine were effective neuroprotectors in the cerebral cortex, and were also effective to reduce hemispheric brain swelling. These amino acids also suppressed glutamate-evoked neuronal activity in vivo. These results suggest that daily ingestion of lysine and/or arginine might be beneficial to prevent cerebral ischemic injury.

\section{ACKNOWLEDGMENTS}

Our research techniques have been developed from those in the Japan Science and Technology Agency (JST). We thank members of Torii Nutrient-stasis Project (1990-1996) on the Exploratory Research for Advanced Technology (ERATO).

I., Zaharchuk, G., Moskowitz, M. A., and Rosen, B. R. (1998). Measurement of changes in cerebral blood volume in spontaneously hypertensive rats following L-arginine infusion using dynamic susceptibility contrast MRI. Magn. Reson. Med. 39, 160-163.

Chang, Y.F., and Gao, X. M. (1995). L-lysine is a barbiturate-like anticonvulsant and modulator of the benzodiazepine receptor. Neurochem. Res. 20, 931-937.

Conigrave, A. D., Quinn, S. J., and Brown, E. M. (2000). L-Amino acid sensing by the extracellular $\mathrm{Ca}^{2+}$-sensing receptor. Proc. Natl. Acad. Sci. USA 97, 4814-4819.

Curtis, D. R., and Koizumi, K. (1961) Chemical transmitter substances in brain stem of cat. J. Neurophysiol. 24, 80-90.

Dalkara, T., Morikawa, E., Panahian, N., and Moskowitz, M. A. (1994). Blood flow-dependent functional recovery in a rat model of focal cerebral ischemia. Am. J. Physiol. 267, H678-H683.

Datta, D., Kundu, P. K., Biswas, S. Dasgupta, S., Bhinge, A., and Chandran, V. (2000). Effect of cationic amino acid, L-lysine and its polymers on the growth and secretion of hybridoma cell line OKT-3. Hybridoma 19, 339-346.

Ebenezar, K. K., Sathish, V., and Devaki, T. (2003). Effect of L-arginine and L-lysine on lysosomal hydrolases and membrane bound phosphatases in 
experimentally induced myocardial infarction in rats. Mol. Cell Biochem. 247, 163-169.

Egan, J.M., Henderson, T.E., and Bernier, M. (1995). Arginine enhances glycogen synthesis in response to insulin in 3T3-L1 adipocytes. Am. J. Physiol. 269, E61-E66.

Halliwell, B., Gutteridge, J. M. C., and Cross, C. E. (1992). Free radicals, antioxidants, and human disease: Where we are now? J. Lab. Clin. Med. $119,598-620$.

Hatashita, S., Hoff, J. T., and Salamat, S. M. (1988). Ischemic brain edema and the osmotic gradient between blood and brain. J. Cereb. Blood Flow Metab. 8, 552-559.

Johnson, J. L. (1978). The excitant amino acids glutamic and aspartic acid as transmitter candidates in the vertebrate central nervous system. Prog. Neurobiol. 10, 155-202.

Kato, T.,Sano, M., and Mizutani, N. (1987). Inhibitory effect of intravenous lysine infusion on urea cycle metabolism. Eur. J. Pediatr. 146, 56-58.

Kondoh, T., Uneyama, H., Nishino, H., and Torii, K. (2002). Melatonin reduces cerebral edema formation caused by transient forebrain ischemia in rats. Life Sci. 72, 583-590.

Kozniewska, E., Roberts, T. P. L., Tsuura, M., Mintorovitch, J., Moseley, M. E., and Kucharczyk, J. (1995). NG-nitro$\mathrm{L}$-arginine delays the development of brain injury during focal ischemia in rats. Stroke 26, 282-289.

Kuluz, J. W., Prado, R. J., Dietrich, W. D., Schleien, C. L., and Watson, B. D. (1993). The effect of nitric oxide synthase inhibition on infarct volume after reversible focal cerebral ischemia in conscious rats. Stroke 24, 2023-2029.

Lewén, A., Matz, P., and Chan, P.H. (2000). Free radical pathways in CNS injury. $J$. Neurotrauma 17, 871-890.

Lipton, S. A., Choi, Y. B., Pan, Z. H., Lei, S. Z., Chen, H, S., Sucher, N. J., Loscalzo, J., Singel, D. J., and Stamler, J.S. (1993).A redox-based mechanism for the neuroprotective and neurodestructive effects of nitric oxide and related nitrosocompounds. Nature 364, 626-632.

Mayer, M. L., Westbrook, G. L., and Guthrie, P. B. (1984). Voltagedependent block by $\mathrm{Mg}^{2}+$ of NMDA responses in spinal cord neurones. Nature 309, 261-263.

Morikawa, E., Rosenblatt, S., and Moskowitz, M. A. (1992). L-Arginine dilates rat pial arterioles by nitric oxide-dependent mechanisms and increases blood flow during focal cerebral ischaemia. Br. J. Pharmacol. 107, 905-907.

Morikawa, E., Moskowitz, M. A., Huang, Z., Yoshida, T., Irikura, K., and Dalkara, T. (1994). L-Arginine infusion promotes nitric oxide-dependent vasodilation, increases $\mathrm{rCBF}$ and reduces infarction volume in the rat. Stroke 25, 429-435.

Nishimura, F., Nishihara, M., Mori, M., Torii, K., and Takahashi, M. (1995). Excitability of neurons in the ventromedial nucleus in rat hypothalamic slices: modulation by amino acids at cerebrospinal fluid levels. Brain Res. 691, 217-222.

Nowak, L., Bregestovski, P., Ascher, P., Herbert, A., and Prochiantz, A. (1984). Magnesium gates glutamate-activated channels in mouse central neurones. Nature 307, 462-465.

Oldendorf, W. H., and Szabo, J. (1976). Amino acid assignment to one of three blood-brain barrier amino acid carriers. Am. J. Physiol. 230, 94-98.

Paxinos, G., and Watson, C. (1997). The Rat Brain in Stereotaxic Coordinates, Compact, 3rd Edn. San Diego: Academic Press.

Quast, M. J., Wei, J., and Huang, N. C. (1995). Nitric oxide synthase inhibitor NG-nitro-L-arginine methyl ester decreases ischemic damage in reversible focal cerebral ischemia in hyperglycemic rats. Brain Res. 677, 204-212.

Ridet, J. L., Malhotra, S. K., Privat, A., and Gage, F. H. (1997). Reactive astrocytes: cellular and molecular cues to biological function. Trends Neurosci. 20, 570-577.

Sadoshima, S., Nagao, T., Okada, Y., Fujii, K., Ibayashi, S., and Fujishima, M. (1997). L-Arginine ameliorates recirculation and metabolic derangement in brain ischemia in hypertensive rats. Brain Res. 744, 246-252.

Satoh, T., and Lipton, S. A. (2007). Redox regulation of neuronal survival mediated by electrophilic compounds. Trends Neurosci. 30, 37-45.

Smriga, M., Ando, T., Akutsu, M., Furukawa, Y., Miwa, K., and Morinaga, Y. (2007). Oral treatment with L-lysine and L-arginine reduces anxiety and basal cortisol levels in healthy humans. Biomed. Res. 28, 85-90.

Smriga, M., Ghosh, S., Mouneimne, Y., Pellett, P. L., and Scrimshaw, N. S. (2004). Lysine fortification reduces anxiety and lessens stress in fam- ily members in economically weak communities in Northwest Syria. Proc. Natl. Acad. Sci. USA 101, 8285-8288.

Smriga, M., and Torii, K. (2003a). L-Lysine acts like a partial serotonin receptor 4 antagonist and inhibits serotoninmediated intestinal pathologies and anxiety in rats. Proc. Natl. Acad. Sci. USA 100, 15370-15375.

Smriga, M., and Torii, K. (2003b). Prolonged treatment with L-lysine and $\mathrm{L}$-arginine reduces stress-induced anxiety in an elevated plus maze. Nutr. Neurosci. 6, 125-128.

Smulders, R.A., Aarsen, M., Teerlink, T., de Vries, P. M., van Kamp, G. J., Donker, A. J., and Stehouwer, C. D. (1997) Haemodynamic and biochemical responses to $\mathrm{L}$-arginine and $\mathrm{L}$-lysine infusions in normal subjects: $\mathrm{L}-\mathrm{ar}-$ ginine induced vasodilation causes to be explained by non-specific effects of cationic amino acids. Clin. Sci. (Colch) 92, 367-374.

Tamura, R., Kondoh, T., Ono, T., Nishijo, H., and Torii, K. (2000). Effects of repeated cold stress on activity of hypothalamic neurons in rats during performance of operant licking task.J. Neurophysiol. 84, 2844-2858.

Torii, K., Mimura, T., and Yugari, Y. (1987). "Biochemical mechanism of umami taste perception and effect of dietary protein on the taste preference for amino acids and sodium chloride in rats," in Umami: A Basic Taste, eds Y Kawamura and M. R. Kare (New York: Marcel Dekker), 513-563.

Torii, K., Uneyama, H., Nishino, H., and Kondoh, T. (2004). Melatonin suppresses cerebral edema caused by middlecerebral artery occlusion/reperfusion in rats assessed by magnetic resonance imaging. J. Pineal Res. 36, 18-24.

Toung, T. J. K., Hurn, P. D., Traystman, R. J., and Bhardwaj, A. (2002). Global brain water increases after experimental focal cerebral ischemia: effect of hypertonic saline. Crit. Care Med. 30, 644-649.

Wellendorph, P., Hansen, K. B., Balsgaard, A., Greenwood, J. R., Egebjerg, J., and Bräuner-Osborne, H. (2005). Deorphanization of GPRC6A: a promiscuous L-alpha-amino acid receptor with preference for basic amino acids. Mol. Pharmacol. 67, 589-597.

White, H. S., Chow, S. Y., Yen-Chow, Y. C. and Woodbury, D. M. (1992). Effect of elevated potassium on the ion content of mouse astrocytes and neurons.
Can. J. Physiol. Pharmacol. 70 (Suppl), S263-S268.

Won, S. J., Kim, D. Y., and Gwag, B. J. (2002). Cellular and molecular pathways of ischemic neuronal death. $J$. Biochem. Mol. Biol. 35, 67-86.

Yamori, Y., Horie, R., Nara, Y., Ohtake, M., and Ikeda, K. (1977). Prophylactic trials for stroke in stroke-prone SHR (2). Effects of fat, protein and amino acids. Jpn. Heart J. 18, 551-553.

Yamori, Y., Horie, R., Ohtaka, M., Nara, Y., and Ikeda, K. (1978). Prophylactic trials for stroke in stroke-prone SHR. (3) Amino acid analysis of various diets and their prophylactic effect. Jpn. Heart J. 19, 624-626.

Young, V. R., and Pellett, P. L. (1990). Current concepts concerning indispensable amino acid needs in adults and their implications for international nutrition planning. Food Nutr. Bull. 12, 289-300.

Zhao, X., Ross, M. E., and Iadecola, C. (2003). L-Arginine increases ischemic injury in wild type mice but not in iNOS-deficient mice. Brain Res. 966 , 308-311.

Zarei, M. M., and Dani, J. A. (1994). Ionic permeability characteristics of the $\mathrm{N}$-methyl-D-aspartate receptor channel. J. Gen. Physiol. 103, 231-248.

Zhang, F., and Iadecola, C. (1994). Reduction of cerebral ischemic damage by delayed treatment with nitric oxide donors. J. Cereb. Blood Flow Metab. 14, 574-580.

Conflict of Interest Statement: The authors declare that the research was conducted in the absence of any commercial or financial relationships that could be construed as a potential conflict of interest.

Received: 03 March 2010; paper pending published: 02 April 2010; accepted: 25 May 2010; published online: 14 June 2010. Citation: Kondoh T, Kameishi M, Mallick HN, Ono T and Torii K (2010) Lysine and arginine reduce the effects of cerebral ischemic insults and inhibit glutamateinduced neuronal activity in rats. Front. Integr. Neurosci. 4:18. doi: 10.3389/ fnint.2010.00018

Copyright (c) 2010 Kondoh, Kameishi, Mallick, Ono and Torii. This is an openaccess article subject to an exclusive license agreement between the authors and the Frontiers Research Foundation, which permits unrestricted use, distribution, and reproduction in any medium, provided the original authors and source are credited. 